\section{Revista Facultad 2020 de Ciencias Básicas Vol. 16(2)}

julio - diciembre

\title{
Fotocatálisis aplicada a la degradación de pesticidas (Profenofos) vertidos en el lago de Tota*
}

\section{Ángela María Rubiano Barrera ${ }^{a}$}

Resumen: este trabajo tiene como objetivo principal evaluar la remoción de un pesticida vertido en el lago de Tota mediante degradación fotocatalítica. El contaminante seleccionado fue Profenofos, principio activo del Awake 500 EC, agroquímico usado en los cultivos de cebolla larga ubicados en la ronda del lago. En la fase experimental, se utilizaron dos métodos de degradación fotocatalítica: suspensión de catalizador $\mathrm{TiO}_{2}$ Degussa P25 e inmovilización de sol-gel de nanopartículas de titanio NTO en vidrio. La fuente de radiación utilizada en laboratorio y a nivel piloto fue una lámpara uv que simula la radiación solar. Se analizó el efecto del tiempo de radiación y la masa de fotocatalizador sobre la concentración del compuesto degradado. Para la determinación analítica de Profenofos se utilizó espectrofotometría uv-Vis y cromatografía de gases con detector N/P y de captura de electrones. El fotocatalizador soportado fue caracterizado con SEM, rayos x e IR. Los resultados obtenidos muestran un alto porcentaje de remoción y reflejan el potencial que la fotocatálisis tiene en la degradación de agroquímicos presentes en aguas del lago de Tota.

Palabras clave: fotocatálisis; lago de Tota; Profenofos; tratamiento de aguas; remoción de pesticidas

Recibido: 22 de septiembre de 2018

Aceptado: 01 de mayo de 2020

Disponible en línea: 27 de agosto de 2021

Cómo citar: B. A. Hurtado Murcia, H. González Sierra, y J. A. Mendoza Suárez, «Generación de las matrices de espín de Paulí a partir de los vectores de Jones», Rev. Fac. Cienc. Básicas, vol. 16, n. 2 2, pp. 77-85, ago. 2021.

* Artículo de investigación.

a Magíster en Diseño de Procesos y Productos, Universidad de los Andes. Ingeniera Química, Universidad Nacional de Colombia, Bogotá, Colombia.

Correo electrónico: am.rubiano@uniandes.edu.co 


\title{
Photocatalysis Applied to the Degradation of Pesticides (Profenofos) dumped in Lake Tota
}

\begin{abstract}
Lake Tota through photocatalytic degradation. The pollutant selected was Profenofos, active principle of Awake 500 EC, an agrochemical used in the long onion crops located around the lake. In the experimental phase, two photocatalytic degradation methods were used: suspension of $\mathrm{TiO}_{2}$ Degussa P25 catalyst and sol-gel immobilization of NTO titanium nanoparticles in glass. The radiation source used in the laboratory and at the pilot was a uv lamp that simulates solar radiation. The effect of radiation time and photocatalyst mass on the concentration of the degraded compound was analyzed. For analytical determination of Profenofos, uv-Vis spectrophotometry and gas chromatography with N/P detector and electron capture were used. The photocatalyst supported was characterized with SEM, X-rays and IR. Results obtained show high percentage of removal and reflect the potential that photocatalysis has in degrading agrochemicals present in the waters of Lake Tota.
\end{abstract}

Keywords: photocatalysis; lake of Tota; Profenofos; water treatment; pesticide removal

\section{Degradação fotocatalítica de pesticidas (Profenofós) vertidos no lago de Tota, Colômbia}

Resumo: o objetivo principal deste trabalho é avaliar a remoção de um pesticida vertido no lago de Tota, Colômbia, mediante degradação fotocatalítica. O contaminante selecionado foi Profenofós, princípio ativo do Awake 500 EC, agroquímico usado nos cultivos de cebola localizados na ribeira do lago. Na fase experimental, foram utilizados dois métodos de degradação fotocatalítica: suspensão de catalisador $\mathrm{TiO}_{2}$ Degussa P25 e imobilização de sol-gel de nanopartículas de titânio NTO em vidro. A fonte de radiação utilizada em laboratório e no nível piloto foi uma lâmpada uv que simula a radiação solar. Foi analisado o efeito do tempo de radiação e da massa de fotocatalisador sobre a concentração do composto degradado. Para a determinação analítica de profenofós, foi utilizada espectrofotometria uV-Vis e cromatografia de gases com detector N/P e de captura de elétrons. $\mathrm{O}$ fotocatalisador suportado foi caracterizado com SEM, raios-x e IR. Os resultados obtidos mostram alta porcentagem de remoção e refletem o potencial que a fotocatálise tem na degradação de agroquímicos presentes na água do lago de Tota.

Palavras-chave: fotocatálise; lago de Tota; Profenofós; tratamento de águas; remoção de pesticidas 


\section{Introducción}

El lago de Tota es un cuerpo de agua natural situado en el departamento de Boyacá, Colombia. Se encuentra ubicado a $15 \mathrm{~km}$ al sur de la ciudad de Sogamoso, en jurisdicción de los municipios de Cuítiva, Tota y Aquitania.

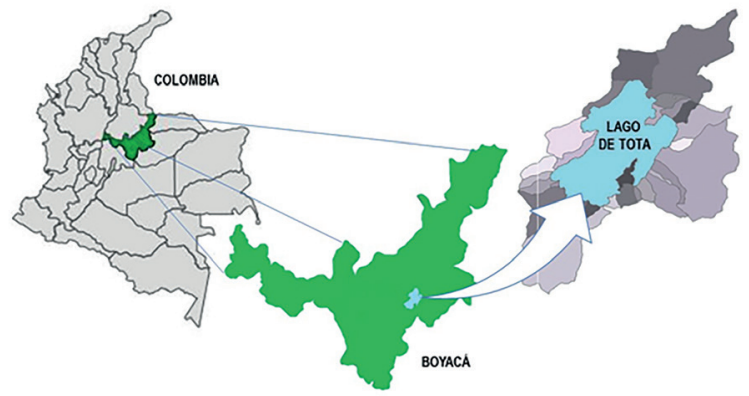

Figura 1. Localización de la cuenca del lago de Tota. Fuente: IGAC.

En el 2012, la World Wetland Network (Red Mundial de Humedales), organización del Reino Unido, le otorgó el premio Globo Gris al lago de Tota por considerar que está bajo amenaza, debido, entre otras causas, al uso de agroquímicos en los cultivos de cebolla larga aledaños al cuerpo lacustre.

Se han identificado como agentes contaminantes principales Propineb, Dimethomorth, Mancozeb, Malathion y Profenofos [1], [2], para los cuales, mediante simulación matemática utilizando modelo Cepis, fueron calculados los valores máximos de descarga en conformidad con los parámetros hídricos del lago, su capacidad asimilativa, el régimen de precipitaciones de la zona, las cantidades aplicadas de agroquímicos y las propiedades fisicoquímicas de los compuestos.

El porcentaje de descarga en el valor límite y el nivel de toxicidad [1] fueron utilizados como criterios de selección del contaminante a degradar. El Profenofos, principio activo del Awake 500 EC, fue el compuesto seleccionado. La concentración de Profenofos calculada mediante el modelo Cepis fue de $0,6 \mu \mathrm{g} / \mathrm{L}$.

La degradación fotocatalitica hace parte de los llamados métodos no convencionales de tratamiento de aguas [4], [5], [10], que han demostrado buenos resultados en la mineralización de moléculas complejas como, por ejemplo, los pesticidas y los fármacos, a sustancias simples y no peligrosas [6], [8], [9]. Estos procesos, al ser combinados con el uso de energías renovables como la luz solar [11], [13], otorgan un importante y significativo valor medioambiental, de manera que se constituye en un claro ejemplo de tecnología sostenible [7]. Estos procesos, al ser combinados con el uso de energías renovables como, por ejemplo, la luz solar, otorgan un importante y significativo valor medioambiental, de modo que se constituyen en un claro ejemplo de tecnología sostenible [7].

La descontaminación de aguas basada en la fotocatálisis solar ha sido empleada desde hace algunos años en diferentes proyectos productivos y de investigación alrededor del mundo, tales como Life-Aquemfree [12] y las plantas piloto de la plataforma solar de Almería, España.

En este sentido, la provincia del Sugamuxi se encuentra en una zona de medio-alto nivel de radiación solar global, de acuerdo con la información registrada en el Atlas de Radiación Solar de Colombia, publicado por la UPME y el Ideam [3]. Esto indica un amplio potencial energético en la región para el desarrollo de sistemas de descontaminación ambiental como lo es la fotocatálisis solar.

\section{Materiales y métodos}

\section{Fase experimental en laboratorio}

La solución madre de Profenofos fue preparada a una concentración de $5 \mathrm{ppm}$, con una muestra comercial de Awake 500 ec (500g de componente activo/L de muestra). El pH fue ajustado en 7,7, de acuerdo con los parámetros suministrados por Corpoboyaca, correspondientes a los monitoreos realizados al lago de Tota durante el último año.

\section{Sistema de fotocatalizador en suspensión}

Datos preliminares fueron obtenidos a partir de la técnica de catalizador en suspensión, para lo cual se utilizó dioxido de titanio Degussa P25 SigmaAldrich diluido en $1 \mathrm{~L}$ de solución madre. 
El sistema utilizado consta de una lámpara compuesta por dos tubos de luz negra UV de $20 \mathrm{~W}$, la cual se irradia sobre una bandeja de aluminio que contiene la solución madre agitada a $500 \mathrm{rpm}$.

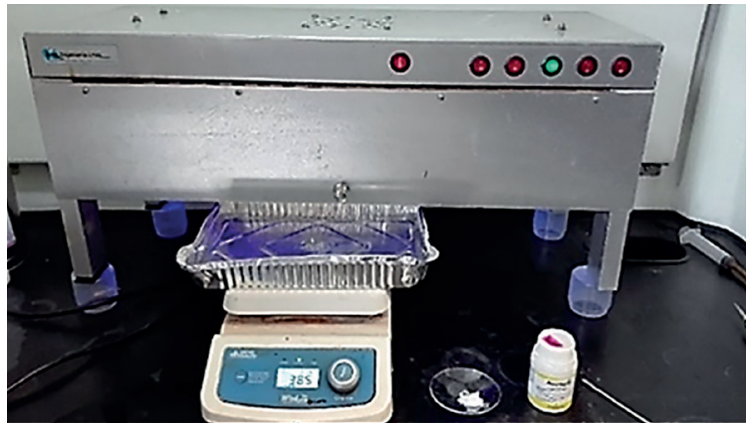

Figura 2. Sistema de fotocatalizador en suspensión. Fuente: elaboración propia.

La variación de la concentración de Profenofos como función del tiempo de irradiación, para diferentes masas de catalizador $(80,120,200,400$, 600 y 1000 mg de Degussa P25), fue determinada usando un espectrofotómetro UV-Vis Thermospectronic Genesys 5, con absorbancia a $277 \mathrm{~nm}$. La determinación analítica se realizó previa extracción líquido-líquido, utilizando diclorometano como solvente de extracción.

La Figura 3 muestra la curva de calibración obtenida usando soluciones de pesticida que contenían de 0,0001 a $10 \mathrm{mg} / \mathrm{L}$ de Profenofos.

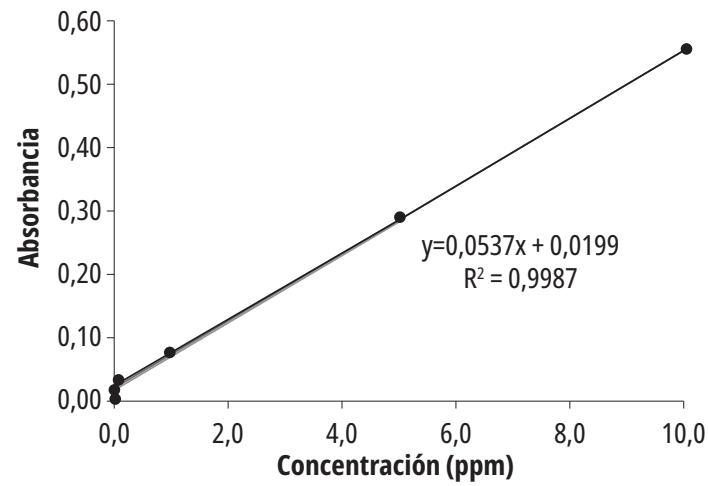

Figura 3. Curva de calibración concentración (ppm) espectro uv-Vis.

Fuente: elaboración propia.

La eficiencia de fotodegradación de Profenofos se calculó de la siguiente manera:

$\%$ Efi.fot. $=(\mathrm{Co}-\mathrm{Cf}) / \mathrm{Co} \times 100$
Donde Co es la concentración inicial de Profenofos, y Cf la concentración final a cierto tiempo de reacción.

\section{Sistema de fotocatalizador soportado}

La preparación de NTO o nanofotocatalizadores de $\mathrm{TiO}_{2}$ se llevó a cabo por el método sol-gel [19]. En este método, $7 \mathrm{ml}$ de isopropóxido de titanio al $97 \%$ se agregaron en $6 \mathrm{ml}$ de etanol absoluto en condiciones de agitación continua durante treinta minutos para obtener solución A. A fin de obtener la solución B, se mezclaron, a $4{ }^{\circ} \mathrm{C}, 0,1 \mathrm{~mL}$ de agua desionizada, $4 \mathrm{~mL}$ de etanol absoluto y $0,132 \mathrm{ml}$ de ácido clorhídrico al 37 \%. Luego, se agregó la solución B gota a gota en la solución A bajo agitación durante cuarenta minutos.

Durante la reacción se agregaron $2 \mathrm{ml}$ de etanol absoluto cada diez minutos, completando $8 \mathrm{ml}$ al final de la agitación.

El gel resultante fue impregnado sobre la superficie del reactor de vidrio y sometido a tratamiento térmico durante cinco horas a $60{ }^{\circ} \mathrm{C}$ y una hora a $500{ }^{\circ} \mathrm{C}$. Este método propone algunas modificaciones al publicado por Lee [14].

El fotocatalizador preparado fue caracterizado mediante microscopía electrónica Microsem, análisis de rayos X-método de difracción DRX y estudio de infrarrojo de $\mathrm{TiO}_{2}$.

En el experimento de planta piloto, el reactor fotoquímico consiste en una estructura cilíndrica de vidrio de $100 \mathrm{~cm} \mathrm{x} 10 \mathrm{~cm}$ (altura $\times$ diámetro), provisto de un sistema de recirculación de $5 \mathrm{~L} / \mathrm{min}$ y un tanque de alimentación con una capacidad de 15 galones. La solución madre es preparada a una concentración de 5 ppm de Profenofos y pH 7,7.

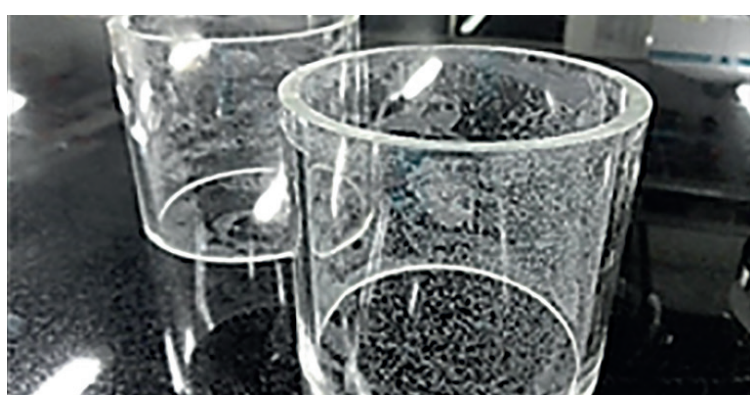

Figura 4. Detalle del sol gel de NTO soportado en el vidrio del reactor fotoquímico.

Fuente: elaboración propia. 


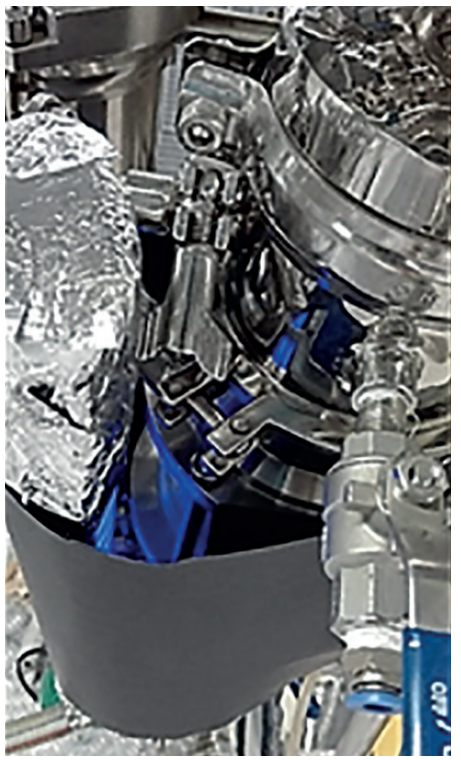

Figura 5. Detalle de la radiación emitida.

Fuente: elaboración propia.

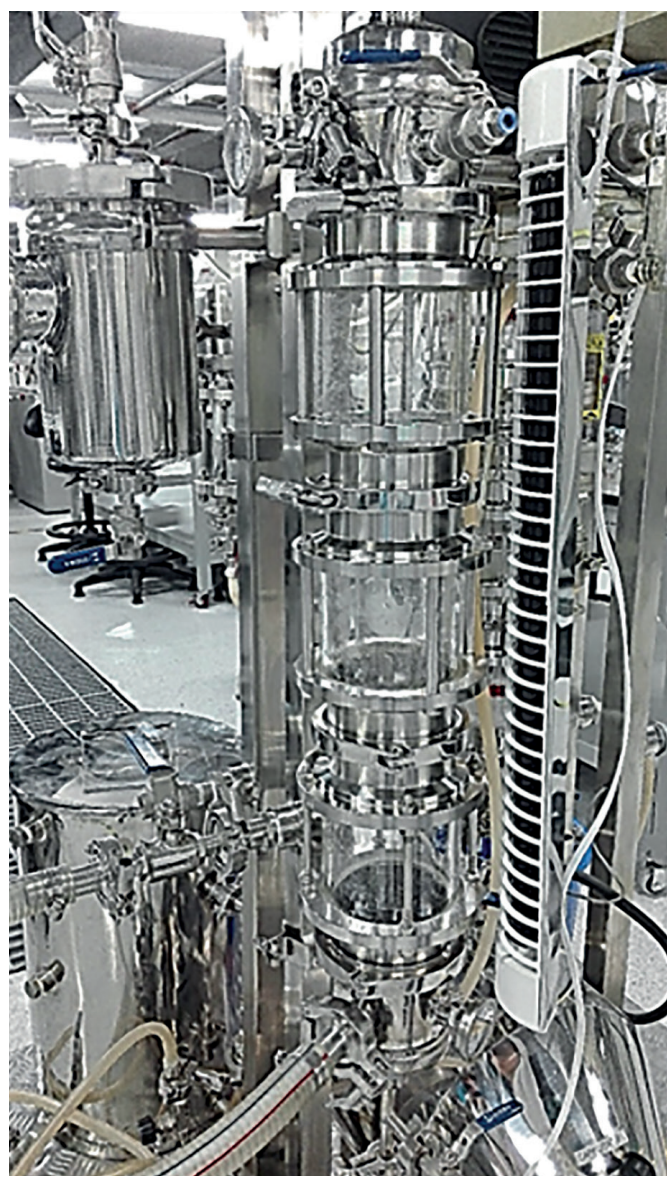

Figura 6. Detalle del reactor fotoquímico. Fuente: elaboración propia.
La fotodegradación se llevó a cabo utilizando una lámpara de luz negra uv de $20 \mathrm{~W}$ y $\Lambda=254 \mathrm{~nm}$, colocada sobre un soporte paralelo al fotorreactor y aislada del exterior por medio de una cubierta negra.

Se tomaron alícuotas cada treinta minutos y se caracterizaron en el espectrofotómetro UV-Vis.

El mejor resultado obtenido durante el seguimiento por espectrofotometría UV-Vis se envío al Laboratorio de Residuos de Plaguicidas de la Universidad Nacional (acreditado por el onAC) para ser analizado mediante cromatografía de gases con detector de electrones y N/P, y confirmar la concentración final.

La eficiencia de la fotodegradación se calcula de acuerdo con la ecuación (1).

\% Efi.fot. $=(\mathrm{Co}-\mathrm{Cf}) / \mathrm{Co} \times 100$

Donde, Co es la concentración inicial de Profenofos, y $C f$ la concentración final a cierto tiempo de reacción.

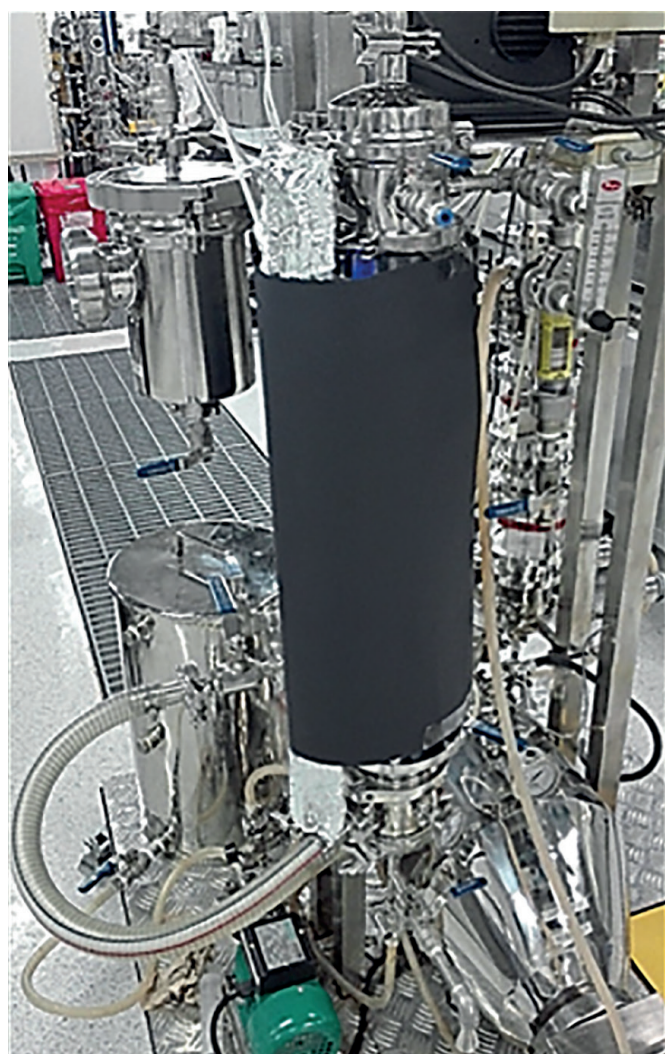

Figura 7. Detalle del reactor fotoquímico con aislamiento. Fuente: Laboratorio de Ingeniería Química Uniandes. 


\section{Resultados y discusión}

Con el fin de realizar un comparativo entre las eficiencias de degradación de Profenofos se estudiaron las técnicas de fotodegradación en suspensión y fotodegradación con NTO soportado.

\section{Fotodegradación en suspensión}

El efecto de la dosis de fotocatalizador sobre la eficiencia de degradación de Profenofos se estudió variando la masa de catalizador en $80,120,200$, 400, 600 y 1000 mg de dióxido de titanio Degussa P25. Se fijó la dosis óptima al observar que la eficiencia de la degradación aumentó hasta en un $96 \%$ en 400 mg para luego volver a disminuir.

$\mathrm{Al}$ incrementar la dosis de $\mathrm{TiO}_{2}$ aumenta también la cantidad de fotones de luz uv y el número

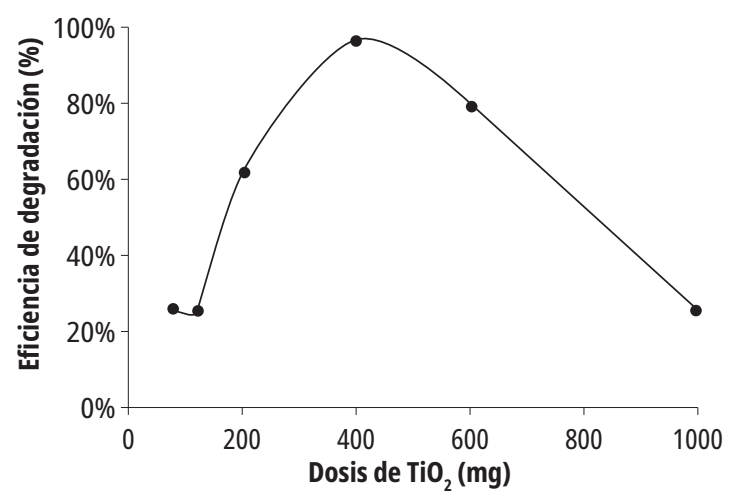

Figura 8. Efecto de la dosis de $\mathrm{TiO}_{2}$ sobre la degradación de Profenofos.

Fuente: elaboración propia.

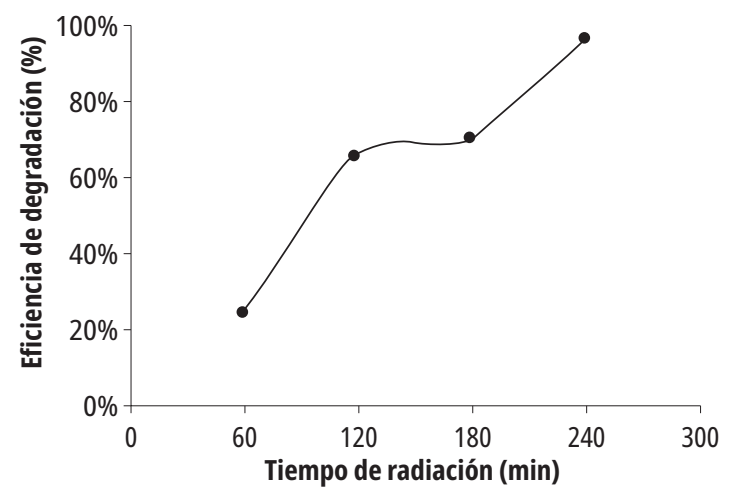

Figura 9. Efecto del tiempo de radiación sobre la degradación de Profenofos para una dosis de $400 \mathrm{mg}$ de fotocatalizador.

Fuente: elaboración propia. de moléculas de pesticida absorbidas en la superficie del catalizador, lo que conduce a un aumento de la tasa de reacción fotocatalítica. Sin embargo, por encima de $400 \mathrm{mg}$ hay una disminución en el área superficial del catalizador debido a la agregación del $\mathrm{TiO}_{2}$, al igual que un posible aumento en la turbidez de la solución, lo cual disminuye la penetración de la luz uv y con ello la fotoactivación [15].

El efecto del tiempo de irradiación sobre la concentración de Profenofos se estudió fijando la masa de catalizador en 400 mg (Figura 9).

\section{Fotodegradación con NTO soportado}

\section{Caracterización fotocatalizador}

La estructura de las nanopartículas de $\mathrm{TiO}_{2}$ (obtenidas por el método de sol-gel) fue caracterizada por difractómetro de rayos $\mathrm{x}$.

Los picos en $25,3,38,0,48,1,54,5,62,8,69,1$ y 75,3 se atribuyen a reflejos de anatasa $\mathrm{TiO}_{2}$, de acuerdo con la tarjeta del Joint Committee on Powder Diffraction Standards (JCPDS n. ${ }^{\circ}$ 21-1272) correspondiente a anatasa $\mathrm{TiO}_{2}[16]$.

Las figuras 10 y 11 muestran el contraste de los resultados obtenidos entre el análisis DRX realizado en la Universidad de los Andes y la tarjeta JCPDS correspondiente.

Las imágenes de microsem de las nanopartículas de $\mathrm{TiO}_{2}$ preparadas (Figuras 12-15) muestran que el material está conformado por una aglomeración de partículas polimorfas (en su mayoría) y esféricas.

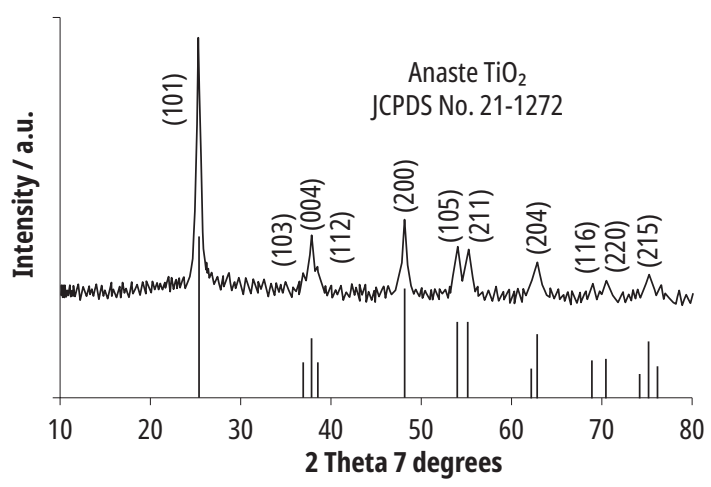

Figura 10. Tarjeta JCPDS n. 21-1272 Anatasa.

Fuente: [18]. 


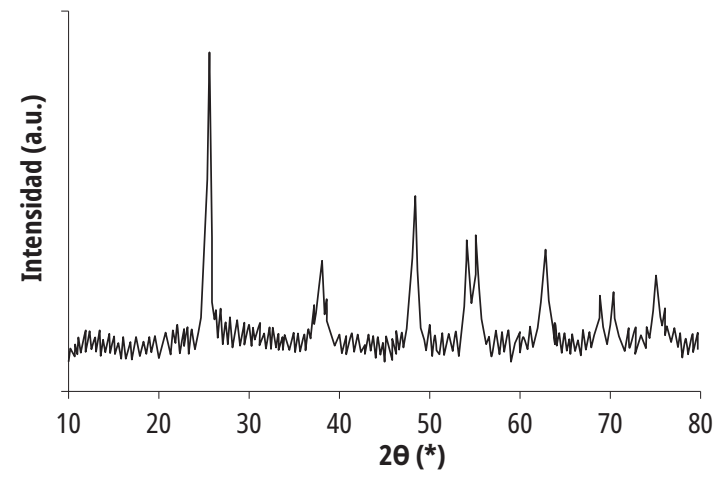

Figura 11. $\mathrm{DRX} \mathrm{TiO}_{2}$. Universidad de los Andes.

Fuente: Centro de Microscopía Uniandes.

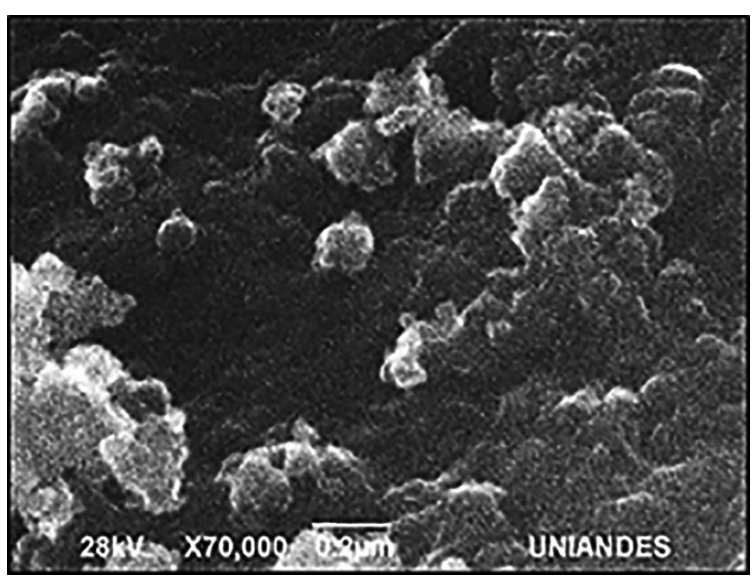

Figura 12. Detalle microsem nanopartículas $\mathrm{TiO}_{2} 0,2 \mu \mathrm{m}$. Fuente: Centro de Microscopía Uniandes.

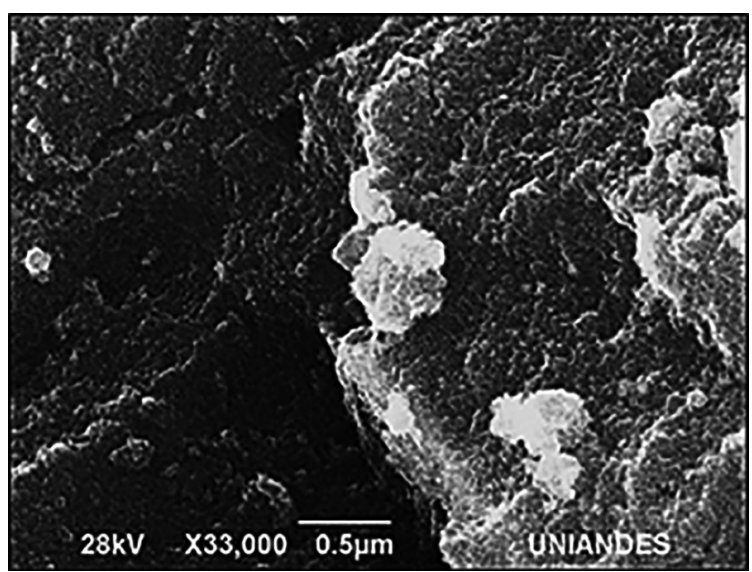

Figura 13. Detalle microsem nanopartículas $\mathrm{TiO}_{2} 0,5 \mu \mathrm{m}$. Fuente: Centro de Microscopía Uniandes.

El software INCA determinó la composición elemental de las nanopartículas. Los resultados se muestran en la Tabla 1.

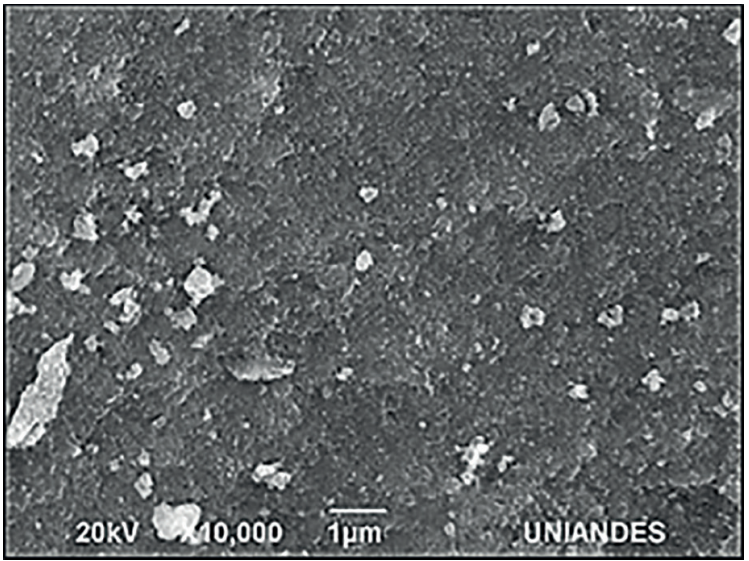

Figura 14. Detalle microsem nanopartículas $\mathrm{TiO}_{2} 1 \mu \mathrm{m}$.

Fuente: Centro de Microscopía Uniandes.

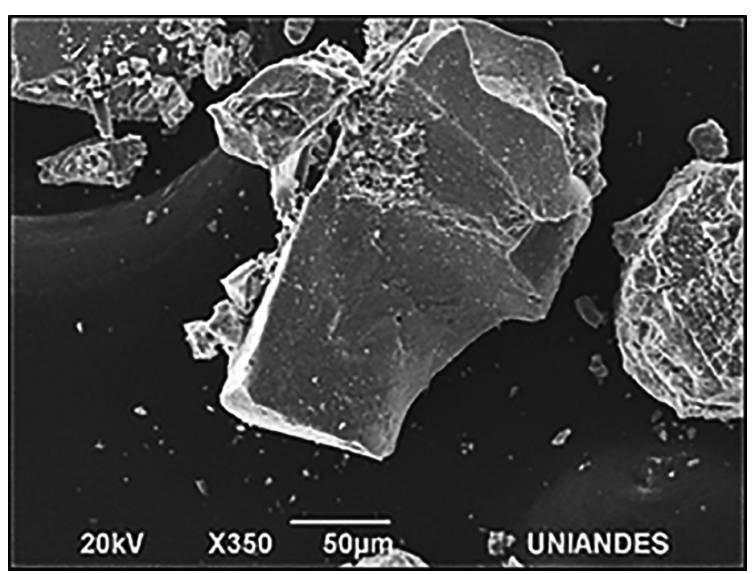

Figura 15. Detalle microsem nanopartículas $\mathrm{TiO}_{2} 50 \mu \mathrm{m}$. Fuente: Centro de Microscopía Uniandes.

Tabla 1. Análisis elemental de las nanopartículas de $\mathrm{TiO}_{2}$

\begin{tabular}{|c|c|c|}
\hline Elemento & \% Peso & \%Atómico \\
\hline 0 & 18,79 & 40,93 \\
\hline $\mathrm{Ti}$ & 81,21 & 59,07 \\
\hline Total & 100 & 100 \\
\hline
\end{tabular}

Fuente: Centro de Microscopía Uniandes.

La Figura 16 muestra el espectro de FTIR de las nanopartículas de $\mathrm{TiO}_{2}$ preparadas.

La banda ancha alrededor 493,8 cm-1 corresponde al modo de flexión Ti-O de $\mathrm{TiO}_{2}$. El ancho de banda observado a $3425,5 \mathrm{~cm}-1$ se debe a las vibraciones generadas por el grupo hidroxilo $(-\mathrm{OH})$ [15]. 


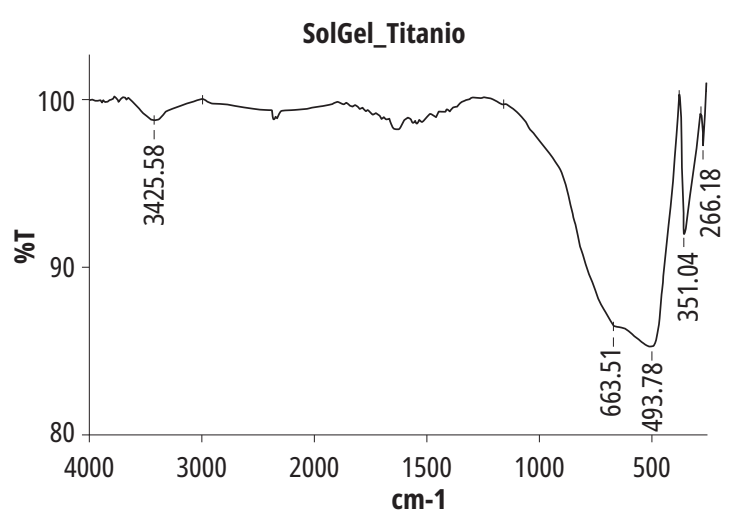

Figura 16. Espectro de FTIR de las nanopartículas de $\mathrm{TiO}_{2}$ preparadas.

Fuente: Laboratorio de Espectroscopía, Facultad de Ciencias, Uniandes.

Valores de anchos de banda cerca a los $660 \mathrm{~cm}-1$ indican vibraciones de torsión del enlace $\mathrm{C}-\mathrm{OH}$ e inferiores a $600 \mathrm{~cm}-1$, la presencia de enlaces bromuro [16].

\section{Conclusiones}

La fotocatálisis con $\mathrm{TiO}_{2}$ demuestra ser efectiva en la degradación de pesticidas presentes en aguas del lago de Tota, como el Profenofos. Un buen complemento de este trabajo consistiría en determinar el ciclo de vida útil del fotocatalizador y la caracterización de los analitos que pueden estar produciéndose durante la fotorreacción. De igual forma, resultaría interesante estudiar el efecto de absorción utilizando otros compuestos, como, por ejemplo, el carbón activado y sus modificaciones.

La eficiencia de degradación fue mayor en el sistema por suspensión (96\%) que en el soportado en vidrio (88 \%); este último fue el sistema seleccionado, teniendo en cuenta el contexto del proyecto, el cual, a escala piloto, se traduce en un fotorreactor constituido por un arreglo de tubos en Pyrex Glass impregnado de nanopartículas de titanio.

El método de impregnación de NTO escogido se basa en la síntesis de un sol-gel. La caracterización evidencia formación de estructura anatasa, la cual teóricamente demuestra mejores resultados frente al rutilo o la brookita. Algunas pérdidas de catalizador en el proceso de secado fueron detectadas, por lo que se sugiere en futuras investigaciones realizar un pretratamiento al soporte con el fin de aumentar su adherencia.

La fuente de radiación utilizada para los ensayos (lámpara Uv) ofrece una idea de la simulación solar. Se sugiere realizar ensayos con luz solar e in situ para verificar los datos.

\section{Agradecimientos}

Agradecimientos a la Dra. Rocío Sierra Ramírez, al Dr. Juan Carlos Moreno Piraján y al Grupo de Investigación de Solidos Porosos y Calorimetría de la Universidad de los Andes, por sus aportes técnicos y logísticos durante la realización de este proyecto.

\section{Referencias}

[1] Y. Pesca, "Seguimiento del comportamiento físico químico de los agroquímicos utilizados en el cultivo de cebolla larga en el entorno cercano al lago de Tota y aplicación del modelo Cepis para evaluar impactos", (tesis de B. Sc.), Univ. Ped. Tec. Col., Boyacá, Colombia, 2015.

[2] I. Grandas, "Identificación de los principales ingredientes activos de los agroquímicos usados en el entorno del Lago de Tota”, Rev. Cine. Ag., vol. 13, pp. 91-106, 2016.

[3] Unidad de Planeación Energética-upme, Instituto de Hidrología, Meteorología y Estudios Ambientales-Ideam, Atlas de Radiación Solar de Colombia. 2015.

[4] C. Geankoplis, Procesos de transporte y operaciones unitarias, $3^{\mathrm{a}}$ ed. Compañía Editorial Continental, 1998.

[5] S. Malato, "Eliminación de contaminantes por fotocatálisis heterogénea”, Deg. Plag., vol. 12, pp. 331-349, 2004.

[6] L. Garcés, "La fotocatálisis como alternativa para el tratamiento de aguas residuales", Rev. Lasallista Inv., vol. 1, pp.83-92, 2003.

[7] C. Cheng, "Heterogeneous photocatalytic oxidation an effective tool for wastewater -a review", St. Wat. Manag. Iss., cap. 9, pp. 219-236, 2012.

[8] M. Gualdrón, "Evaluación del potencial de desinfección de agua cruda mediante el aprovechamiento de la energía solar térmica y fotónica a través de un colector cilindro parabólico (СС)", En Simposio Latinoamericano de Procesos Avanzados de Oxidación en Sistemas de Potabilización y Tratamiento de Aguas Residuales, Cali, 2006. 
[9] D. Angthararuk, "Degradation products of profenofos as identified by high-field FTICR mass spectrometry: isotopic fine structure approach", Journal of Environmental Science and Health, part B, vil. 52, n. ${ }^{\circ}$, pp.1022, 2016.

[10] M. Regina, "Photocatalytic degradation of an organophosphorus pesticide from agricultural waste by immobilized $\mathrm{TiO}_{2}$ under solar radiation”, Amb. \& Ág., vol. 11, n. ${ }^{\circ}$ 4, pp. 778-787, 2016.

[11] J. Guerrero, “Comparación de dos metodologías para la determinación de residuos de plaguicidas en agua potable”, Rev. Col. Quím., vol. 43, n. ${ }^{\circ}$ 1, pp. 17-24, 2014.

[12] N.V.S. Venugopal, "Uv-Spectrophotometric determination of Acephate and Profenofos (organophosphate) pesticides in buffer medium", As. J. Res. Chem, vol. 5, n. ${ }^{\circ} 4$, pp. 526-528, 2012.

[13] M. Saber, "Influence of parameters on the heterogeneous photocatalytic degradation of pesticides and phenolic contaminants in wastewater", J. Env. Manag., vol. 92, pp. 311-330, 2011.

[14] M. Lazar, "Photocatalytic water treatment by titanium dioxide: recent updates”, Catal., vol. 2, n. $\left.{ }^{\circ} 4\right)$, pp. 572$601,2012$.
[15] P. Fernández-Ibáñez, "Decontamination and disinfection of water by solar photocatalysis: the pilot plants of the Plataforma Solar de Almería”, J Mat. Sci. Semic. Proces., vol. 42, pp.15-23, 2015.

[16] Instituto Murciano de Investigación y Desarrollo Agrario y Alimentario-Imida, Proyecto Life-Aquemfree: Descontaminación en finca de Aguas residuales con productos fitosanitarios procedentes de remanentes, enjuagues y limpiezas mediante fotocatálisis solar. España, 2018. [En línea]. Disponible en http://www. life-aquemfree.eu/.

[17] V. Vílar, "Solar photocatalysis of a recalcitrant coloured effluent from a wastewater treatment plant", Photoch. \& Photob. Sci., vol. 5, 2009.

[18] F. Rojo, “Tablas de Espectroscopia Infrarroja”, Universidad Nacional Autónoma de México, México, 2015. [En línea]. Recuperado de http://depa.fquim.unam. mx/amyd/archivero/TablasIR_34338.pdf

[19] S. Lee, "Synthesis of $\mathrm{TiO}_{2}$ photocatalyst thin film by solvothermal method with a small amount of water and its photocatalytic performance", J. Photochem. Photob. Chem., vol. 146, pp.121-128, 2001. 
